\title{
Linear-correction Extended Kalman Filter for Target Tracking Using TDOA and FDOA Measurements
}

\author{
Bing Deng ${ }^{1,2, *}$, He Qin ${ }^{2}$, Zhengbo Sun ${ }^{2}$ \\ ${ }^{1}$ Zhengzhou Institute of Information Science and Technology, Zhengzhou, Henan, 86-450002, China. \\ ${ }^{2}$ National Key Laboratory of Science and Technology on Blind Signal Processing, Chengdu, Sichuan, 86-610041, China \\ ${ }^{*}$ Corresponding author
}

\begin{abstract}
This paper considers the target tracking based on TDOA (Time Difference of Arrival) and FDOA (Frequency Difference of Arrival) measurements for a single target in a distributed sensor network. A linear-correction EKF algorithm that involves closed-form weighted least squares (WLS) optimization only is developed to solve the divergence problem of the extended kalman filter (EKF) in performing estimation of the state of a nonlinear system. The proposed method firstly obtains initial state estimation result by EKF using the WLS approach and subtracting its state error, then redoes the filtering process again to overcome the error causing by the part linearization operation for measurement matrix in EKF. The specific expressions TDOA-FDOA-based is provided at last. Simulation results demonstrate the good performance of the proposed method.
\end{abstract}

Keywords-component; target tracking; extended kalman filter (EKF); time difference of arrival (TDOA); frequency difference of arrival (FDOA); linear-correctio

\section{INTRODUCTION}

In recent years, some target positioning and tracking system using TDOA (Time Difference of Arrival) and FDOA (Frequency Difference of Arrival) measurements received at multiple sensors in distributed sensor network consisting of several passive sensors are widely studied[1-6]. Moving source localization and tracking using TDOAs and FDOAs is a challenging problem because of the high nonlinearity inherent in the measurement equations [3-5]. Extended Kalman filter (EKF) is known to be the well-known nonlinear estimator for systems with white target state and measurement noises [7-13]. Its efficiency and ease of implementation have made this estimator an essential tool for a wide class of applications such as signal processing, target tracking, sonar, navigation, wireless communication, and so on. But the EKF is easy to encounter divergence problem caused by part linearing using first-order taylor-series in nonlinear system [10-13].

In this paper, we begin our study with deriving the estimate error of the processing of the EKF method [13] under Gaussian noise model. We then propose a new linearcorrection moving source tracking solution by estimating and subtracting out the EKF estimation error. The new solution is shown to be able to attain better accuracy, compared with benchmark method EKF in [13].

\section{PROBLEM FORMULATION}

Assume there are $m$ moving sensors with positions $\boldsymbol{s}_{i}=\left[x_{i}, y_{i}, z_{i}\right]^{T}, i=1,2, \ldots, m$. The unknown moving source position and velocity is defined as $\boldsymbol{u}_{k}=[x(k), y(k), z(k)]^{T}$ and $\dot{\boldsymbol{u}}_{k}=[\dot{x}(k), \dot{y}(k), \dot{z}(k)]^{T}$ at $k$ discrete-time respectively.

The true range and true range rate between the source and receiver $i$ are

$$
r_{i}^{o}(k)=\left\|\boldsymbol{u}_{k}-\boldsymbol{s}_{i}\right\|
$$

$$
\dot{r}_{i}^{o}(k)=\left(\boldsymbol{u}_{k}-\boldsymbol{s}_{i}\right)^{T}\left(\dot{\boldsymbol{u}}_{k}-\dot{\boldsymbol{s}}_{i}\right) / r_{i}^{o}, i=1,2, \ldots, m
$$

Let $\boldsymbol{x}_{k}=\left[\boldsymbol{u}_{k}^{T}, \dot{\boldsymbol{u}}_{k}^{T}\right]^{T}$. Without loss of generality, we set the first sensor as the reference sensor and denote the true true range and true range rate between the sensor $i$ and 1 as $r_{i 1}^{o}(k)$ and $\dot{r}_{i 1}^{o}(k)$, which are equal to

$$
\begin{aligned}
& r_{i 1}^{o}(k)=r_{i}^{o}(k)-r_{1}^{o}(k)=r_{i 1}(k)+n_{i 1}(k) \\
& \dot{r}_{i 1}^{o}(k)=\dot{r}_{i}^{o}(k)-\dot{r}_{1}^{o}(k)=\dot{r}_{i 1}(k)+\dot{n}_{i 1}(k)
\end{aligned}
$$

where $r_{i 1}(k)$ and $\dot{r}_{i 1}(k)$ are the TDOA and FDOA measurements with noises $n_{i 1}(k)$ and $\dot{n}_{i 1}(k)$. Collecting measurements together, we have

$$
\boldsymbol{\alpha}=\left[\boldsymbol{r}^{o T}, \dot{\boldsymbol{r}}^{o T}\right]^{T}, \Delta \boldsymbol{\alpha}=\left[\boldsymbol{n}^{T}, \dot{\boldsymbol{n}}^{T}\right]^{T}
$$

where $\boldsymbol{r}=\boldsymbol{r}^{o}+\boldsymbol{n}, \dot{\boldsymbol{r}}=\dot{\boldsymbol{r}}^{o}+\dot{\boldsymbol{n}} . \boldsymbol{r}^{o}=\left[r_{21}^{o}(k), r_{31}^{o}(k), \ldots, r_{m 1}^{o}(k)\right]^{T}$, $\dot{\boldsymbol{r}}^{o}=\left[\dot{r}_{21}^{o}(k), \dot{r}_{31}^{o}(k), \ldots, \dot{r}_{m 1}^{o}(k)\right]^{T} . \boldsymbol{n}=\left[n_{21}(k), n_{31}(k), \ldots, n_{m 1}(k)\right]^{T}$ a nd $\dot{\boldsymbol{n}}=\left[\dot{n}_{21}(k), \dot{n}_{31}(k), \ldots, \dot{n}_{m 1}(k)\right]^{T} . \Delta \boldsymbol{\alpha}$ is noise vector, which is zero-mean Gaussian with covariance matrix $\boldsymbol{R}_{k}$. 


\section{ESSENTIALS OF EKF MECHANIZATION}

The standard nonlinear dynamical system for which Extended Kalman-type filters are designed has a discrete-time representation consisting of an $n$-dimensional state vector $\boldsymbol{x}_{k}$ and an $\boldsymbol{l}$-dimensional measurement vector $\boldsymbol{z}_{k}$ of the following well-known form as [12]:

$$
\begin{gathered}
\boldsymbol{x}_{k}=\boldsymbol{f}\left(\boldsymbol{x}_{k-1}\right)+\boldsymbol{w}_{k-1} \\
\boldsymbol{z}_{k}=\boldsymbol{h}\left(\boldsymbol{x}_{k}\right)+\boldsymbol{v}_{k}
\end{gathered}
$$

where $\boldsymbol{f}(\cdot)$ is the known nonlinear state transition matrix, $\boldsymbol{w}_{k-1}$ is system error. $\boldsymbol{h}(\cdot)$ is the nonlinear measurement matrix, $\boldsymbol{v}_{k}$ is the measurement error. There, $\boldsymbol{w}_{k-1}$ and $\boldsymbol{v}_{k}$ are assumed to be independent, identically distributed Gaussian random variables with $\boldsymbol{w}_{k-1} \square N\left(0, \boldsymbol{Q}_{k-1}\right)$ and $\boldsymbol{v}_{k} \square N\left(0, \boldsymbol{R}_{k}\right)$ respectively. The initial condition $\boldsymbol{x}_{0} \square N\left(\overline{\boldsymbol{x}}_{0}, P_{0}\right)$ (Gaussianly distributed, with known mean $\overline{\boldsymbol{x}}_{0}$ and covariance matrix $P_{0}$ ). The three symmetric matrices $P_{0}$ and $\boldsymbol{Q}_{k-1}$ must be positive semidefinite and $\boldsymbol{R}_{k}$ must usually be positive definite. x(0) - $\operatorname{Af}(\mathrm{T}(\mathrm{O}), \mathrm{P}(\mathrm{O})$ ) (Gaussianly covariance matrix $\boldsymbol{Q}_{k}$.

EKF algorithm is a recursive estimation process by consecutively using prediction and updating operation with external measurements. This is accomplished by linearizing(as the first two terms of a Taylor series expansion, involving a constant term and a 1st derivative term, known as the Jacobian) of either the system equation or measurement equation (or both if each is nonlinear) as evaluated about the current state estimate. The recursive process of AKF is as follows

- $\quad$ Step 1: Initializing $\hat{\boldsymbol{x}}_{0}, \boldsymbol{P}_{0}, \boldsymbol{Q}_{0}$ and $\boldsymbol{R}_{0}$.

- $\quad$ Step 2: Calculating state Jacobian.

$$
\boldsymbol{F}_{k}=\left.\frac{\partial \boldsymbol{f}(\boldsymbol{x})}{\partial \boldsymbol{x}}\right|_{\boldsymbol{x}=\hat{\boldsymbol{x}}_{k-1}}
$$

- $\quad$ Step 3:Prediction state.

$$
\hat{\boldsymbol{x}}_{k \mid k-1}=\boldsymbol{f}\left(\hat{\boldsymbol{x}}_{k-1}\right)
$$

- $\quad$ Step 4: Calculating variance of prediction state.

$$
\boldsymbol{P}_{k \mid k-1}=\boldsymbol{F}_{k} \boldsymbol{P}_{k-1} \boldsymbol{F}_{k}^{T}+\boldsymbol{Q}_{k-1}
$$

- $\quad$ Step 5: Calculating prediction residual:

$$
\boldsymbol{Y}=\boldsymbol{z}_{k}-\hat{\boldsymbol{z}}_{k \mid k-1}=\boldsymbol{z}_{k}-\boldsymbol{h}\left(\hat{\boldsymbol{x}}_{k \mid k-1}\right)
$$

- $\quad$ Step 6: Calculating Variance of prediction residual:

$$
\boldsymbol{S}_{k}=\boldsymbol{H}_{k} \boldsymbol{P}_{k \mid k-1} \boldsymbol{H}_{k}^{T}+\boldsymbol{R}_{k}
$$

- $\quad$ Step 7: Calculating Gain matrix:

$$
\boldsymbol{K}_{k}=\boldsymbol{P}_{k \mid k-1} \boldsymbol{H}_{k}^{T} \boldsymbol{S}_{k}^{-1}
$$

- $\quad$ Step 8: Calculating Estimated state:

$$
\hat{\boldsymbol{x}}_{k}=\hat{\boldsymbol{x}}_{k \mid k-1}+\boldsymbol{K}_{k}\left(\boldsymbol{z}_{k}-\hat{\boldsymbol{z}}_{k \mid k-1}\right)
$$

- $\quad$ Step 9: Covariance estimation Variance of estimated state:

$$
\boldsymbol{P}_{k}=\boldsymbol{P}_{k \mid k-1}-\boldsymbol{K}_{k} \boldsymbol{H}_{k} \boldsymbol{P}_{k \mid k-1}
$$

\section{PROPOSED METHOD BASED ON LINEAR-CORRECTION}

Suppose the EKF processing outputs $\hat{\boldsymbol{x}}_{k}=\left[\hat{\boldsymbol{u}}^{T}, \hat{\dot{\boldsymbol{u}}}^{T}\right]^{T}$ that consists of initial estimates of the source position and velocity [3]. Then, $\Delta \boldsymbol{x}_{k}=\left[\Delta \hat{\boldsymbol{u}}^{T}, \Delta \hat{\dot{\boldsymbol{u}}}^{T}\right]^{T}$, and we have $\boldsymbol{u}=\hat{\boldsymbol{u}}-\Delta \boldsymbol{u}$, $\dot{\boldsymbol{u}}=\hat{\dot{\boldsymbol{u}}}-\Delta \dot{\boldsymbol{u}}$, where the $\Delta \boldsymbol{u}$ and $\Delta \dot{\boldsymbol{u}}$ are the estimation errors in $\hat{\boldsymbol{u}}$ and $\hat{\boldsymbol{u}}$ to be identified in the following linear correction step. Applying the Taylor-Series expansion up to first-order terms, we have

$$
\boldsymbol{z}_{k}=\boldsymbol{h}\left(\hat{\boldsymbol{x}}_{k}-\Delta \boldsymbol{x}_{k}\right)+\boldsymbol{v}_{k} \approx \boldsymbol{h}\left(\hat{\boldsymbol{x}}_{k}\right)-\boldsymbol{H}_{k} \Delta \boldsymbol{x}_{k}
$$

Where $\boldsymbol{H}_{k}=\left.\frac{\partial \boldsymbol{h}(\boldsymbol{x})}{\partial \boldsymbol{x}}\right|_{\boldsymbol{x}=\hat{x}_{k}}$. So, re-writing (15), we have

$$
\boldsymbol{z}_{k}-\boldsymbol{h}\left(\hat{\boldsymbol{x}}_{k}\right)+\boldsymbol{H}_{k} \Delta \boldsymbol{x}_{k} \approx \boldsymbol{v}_{k}
$$

$\Delta \boldsymbol{x}_{k}$ can be estimated by the weighted least-squares (WLS) method whose solution is

$$
\Delta \boldsymbol{x}_{k}=-\left(\boldsymbol{H}_{k}^{T} \boldsymbol{W} \boldsymbol{H}_{k}\right)^{-1} \boldsymbol{H}_{k}^{T} \boldsymbol{W}\left[\boldsymbol{z}_{k}-\boldsymbol{h}\left(\hat{\boldsymbol{x}}_{k}\right)\right]
$$

where $\boldsymbol{W}$ is the weighting matrix chosen as $\boldsymbol{W}=(\boldsymbol{R})^{-1}$.

The proposed linear correction technique finds the final state solution $\boldsymbol{x}_{k}$ via updating $\hat{\boldsymbol{x}}_{k}$ using

$$
\boldsymbol{x}_{k}=\hat{\boldsymbol{x}}_{k}-\Delta \boldsymbol{x}_{k}
$$


So, adding the linear-correction processing to the EKF, we can get the linear-correction EKF algorithm.

Now, the expression of the linear-correction TDOA-FDOA based on is

$$
\begin{gathered}
r_{i}^{o}(k) \approx \hat{r}_{i}(k)-\boldsymbol{\rho}_{\hat{u}_{k}, s_{i}}^{T} \Delta \boldsymbol{u}_{k} \\
\dot{r}_{i}^{o}(k) \approx \hat{\dot{r}}_{i}(k)-\boldsymbol{g}_{i}^{T}(k) \Delta \boldsymbol{u}_{k}-\boldsymbol{\rho}_{\hat{u}_{k}, s_{i}}^{T} \Delta \dot{\boldsymbol{u}}_{k}
\end{gathered}
$$

where $\hat{r}_{i}(k)=\left\|\hat{\boldsymbol{u}}_{k}-\boldsymbol{s}_{i}\right\|, \hat{\dot{r}}_{i}(k)=\left(\hat{\boldsymbol{u}}_{k}-\boldsymbol{s}_{i}\right)^{T}\left(\hat{\dot{\boldsymbol{u}}}_{k}-\hat{\boldsymbol{s}}_{i}\right) / \hat{r}_{i}(k), i=1,2, \ldots, m$. $\boldsymbol{\rho}_{\hat{\boldsymbol{u}}_{k}, \boldsymbol{s}_{i}}=\left(\hat{\boldsymbol{u}}_{k}-\boldsymbol{s}_{i}\right) /\left\|\hat{\boldsymbol{u}}_{k}-\boldsymbol{s}_{i}\right\|, \boldsymbol{g}_{i}^{T}(k)=\left(\hat{\dot{\boldsymbol{u}}}_{k}-\dot{\boldsymbol{s}}_{1}\right)^{T}\left(\boldsymbol{I}-\boldsymbol{\rho}_{\hat{u}_{k}, \boldsymbol{s}_{1}} \boldsymbol{\rho}_{\hat{\boldsymbol{u}}_{k}, s_{1}}^{T}\right) / \hat{r}_{1}(k)$.

The measurement matrix $\boldsymbol{h}\left(\hat{\boldsymbol{x}}_{k}\right)$ and $\boldsymbol{H}_{k}$ are

$$
\begin{gathered}
\boldsymbol{h}\left(\hat{\boldsymbol{x}}_{k}\right)=\left[\begin{array}{c}
\hat{r}_{2}(k)-\hat{r}_{1}(k) \\
\vdots \\
\hat{r}_{m}(k)-\hat{r}_{1}(k) \\
\hat{\dot{r}}_{2}(k)-\hat{\dot{r}}_{1}(k) \\
\vdots \\
\hat{\dot{r}}_{m}(k)-\hat{\dot{r}}_{1}(k)
\end{array}\right] \\
\boldsymbol{H}_{k}=\left[\begin{array}{cc}
\boldsymbol{\rho}_{\hat{u}_{k}, s_{2}}^{T}-\boldsymbol{\rho}_{\hat{u}_{k}, s_{1}}^{T} & \boldsymbol{0}^{T} \\
\vdots & \vdots \\
\boldsymbol{\rho}_{\hat{u}_{k}, s_{m}}^{T}-\boldsymbol{\rho}_{\hat{u}_{k}, s_{1}}^{T} & \boldsymbol{0}^{T} \\
\boldsymbol{g}_{2}^{T}(k)-\boldsymbol{g}_{1}^{T}(k) & \boldsymbol{\rho}_{\hat{u}_{k}, s_{i}}^{T}-\boldsymbol{\rho}_{\hat{u}_{k}, s_{1}}^{T} \\
\vdots & \vdots \\
\boldsymbol{g}_{m}^{T}(k)-\boldsymbol{g}_{1}^{T}(k) & \boldsymbol{\rho}_{\hat{u}_{k}, s_{i}}^{T}-\boldsymbol{\rho}_{\hat{u}_{k}, s_{1}}^{T}
\end{array}\right]
\end{gathered}
$$

Now, we finish the derivation of the linear-correction EKF for target tracking using TDOA and FDOA measurements.

\section{SIMULATIONS}

This section provides the performance comparison of the proposed method with the EKF algorithm in [13] via simulations. The simulation scenario contains 2 sensors, whose true positions are listed in Table 1 . The TDOA noise power is $\sigma_{r}^{2}$ and the FDOA noise power is $\sigma_{f}^{2}=0.1 \sigma_{r}^{2}, \sigma_{r}=0.1 \mathrm{~m}$. The covariance matrix of the TDOA and FDOA measurement vector is $\boldsymbol{R}_{k}=\operatorname{diag}\left(\boldsymbol{R}_{t}, \boldsymbol{R}_{f}\right)$, where $\boldsymbol{R}_{t}$ is a $(m-1) \times(m-1)$ matrix with $\sigma_{r}^{2}$ being its diagonal elements and $0.5 \sigma_{r}^{2}$ being its off-diagonal elements. $\boldsymbol{R}_{f}=\sigma_{f}^{2} \boldsymbol{R}_{t}$. The estimation accuracy is assessed by the root mean-square error (RMSE) as [4], which is defined as $\sqrt{\sum_{l=1}^{L}\left\|\hat{\boldsymbol{p}}_{l}-\boldsymbol{p}\right\|^{2} / L}$, where $\hat{\boldsymbol{p}}_{l}$ is the estimate of $\boldsymbol{p}$ at the $l$ th ensemble run, $\boldsymbol{p}$ is the true source position or velocity, and $L=500$ is the total number of Monte Carlo runs.

The simulation scenarios is considered that the source located at $\boldsymbol{u}=[600,650,550]^{T} \mathrm{~m}$ with a velocity of $\dot{\boldsymbol{u}}=[40,-30,10]^{T} \mathrm{~m} / \mathrm{s}$.

TABLE I. TRUE POSITIONS OF THE RECEIVERS

\begin{tabular}{|c|c|c|c|}
\hline receiver no. $i$ & $x_{i}$ & $y_{i}$ & $z_{i}$ \\
\hline 1 & 300 & 100 & 150 \\
2 & 400 & 150 & 100 \\
\hline
\end{tabular}



FIGURE I. RMSE FOR POSITION ESTIMATE

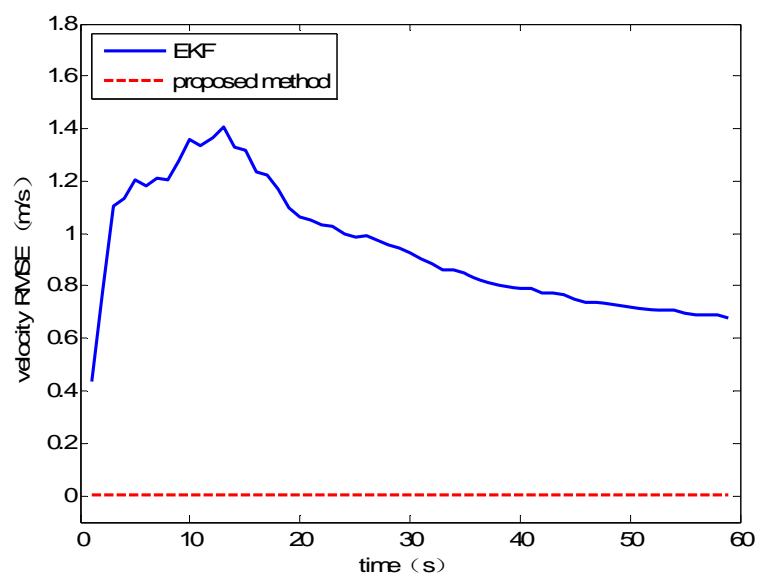

FIGURE II. RMSE FOR VELOCITY ESTIMATE

Figure I and Figure II show the estimates RMSE results for the target position and velocity respectively. It can be shown that the linear-correction method is able to attain a precise tracking accuracy. More importantly, it has better estimation performance than that of the EKF methods at the same conditions. 
The performance improvement come from the formulation of the linear correction step of the new algorithm that involves neglecting second-order error terms only. This is significantly different from commonly adopted Taylor-series method in EKF [8] that require ignoring both second-order and higher-order error terms.

\section{CONCLUSION}

Based on the linear-correction idea, a new TDOA and FDOA moving source tracking algorithm by EKF was developed. The proposed method corrects the initial source tracking result which is obtained in the EKF method by estimating its error. Simulation results demonstrated the good performance of the proposed method.

\section{REFERENCES}

[1] C. Yalu, P. Li, L. Jinzhou, Y. Le and G. Fucheng. "A new iterative algorithm for geolocating a known altitude target using TDOA and FDOA measurements in the presence of satellite location uncertainty," Chinese Journal of Aeronautics, vol.28, no.5,pp.1510-1518, 2015.

[2] L. Jinzhou, G. Fucheng, Y. Le, J. Wenli, and P. Hongwei. "On the use of calibration sensors in source localization using TDOA and FDOA measurements,” Digital Signal Processing, no.27, pp.33-43, 2014

[3] S Ming and Ho K.C.. "An asymptotically efficient estimator for TDOA and FDOA positioning of multiple disjoint sources in the presence of sensor location uncertainties,” IEEE Trans. Signal Process., vol.59, no.7, pp. 3434-3440, 2011

[4] Deng Bing,Sun Zhengbo,Yang Le,Xiong Jinyu. “Geolocation of a known altitude object using TOA measurements,” Journal of Xidian University vol.44, no.3,pp.142-147, 2017.

[5] Z. Guohui, F. Dazheng, X. Hui and Z. Yan. “An approximately efficient bi-iterative method for source position and velocity estimation using TDOA and FDOA measurements,” Signal Processing, no.125, pp.110121, 2016

[6] D. Hu, H. Zhen, C. Xi and L. Jianhua. "A moving source localization method using TDOA, FDOA and Doppler rate measurements,” IEICE Trans. Commun., vol.E99-B, no.3, pp.758-766, 2016

[7] Li Liangqun,Ji Hongbing,Luo Junhui. “Iterated extended kalman particle filtering,” Journal of Xidian University, vol.34, no.2,pp.233-238, 2017.

[8] Liu Y.H.,Li T.,Yang Y.Y.. "Estimation of tire-road friction coefficient based on combined APF-IEKF and iteration algorithm," Mechanical Systems and Signal Processing,vol.88,pp. 25-35,2017.

[9] Yang Yongjian,Fan Xiaoguang,Zhou Zhenfu. “Amended kalman filter for maneuvering target tracking," Chinese Journal of Electronics, vol.25,no.6,pp.1166-1171,2016.

[10] Chen Xiyuan,Xu Yuan,Li Qinghua. "Improving ultrasonic-based seamless navigation for indoor mobile robots utilizing EKF and LSSVMM,” Measurement,vol.92,pp.243-251,2016.

[11] Yongjian Yang, Xiaoguang Fan, Zhenfu Zhuo,et al. “AFAKF for manoeuvring target tracking based on current statistical model,”IET Science, Measurement \& Technology,vol.10,no.6,pp.637-343,2016.

[12] Yongjian Yang, Xiaoguang Fan, Zhenfu Zhuo,et al. “Amended Kalman Filter for Maneuvering Target Tracking,” Chinese Journal of Electronics,vol.25,no.6,pp.1166-1171,2016.

[13] Yuki TAKABAYASHI,Takashi MATSUZAKI,Hiroshi KAMEDA and Masayoshi ITO."Target Tracking Using TDOA/FDOA Measurements in the Distributed Sensor Network,"SICE Annual Conference 2008,August 20-22, 2008, The University Electro-Communications, Japan,pp.34413446. 